\title{
TRATAMENTO CIRÚRGICO DOS ANEURISMAS NÃO ROTOS DA ARTÉRIA CEREBRAL MÉDIA
}

\author{
Luiz Carlos M endes Faleiro ${ }^{1}$, Newton José Godoy Pimenta², Rodrigo M oreira Faleiroº ${ }^{3}$ Rodrigo Antônio \\ Costa ${ }^{2}$, Augusto César Esmeraldo²
}

\begin{abstract}
RESUM 0 - Descrevemos nossa experiência no tratamento microcirúrgico de 34 pacientes portadores de aneurismas não rotos da artéria cerebral média. Houve prevalência no sexo masculino. Oitenta e três por cento dos aneurismas apresentava tamanho menor que $10 \mathrm{~mm}$. Não houve óbito na presente série, com morbidade de 3\% após 1 ano. Indicamos, portanto, microcirurgia como tratamento de escolha para os aneurismas não rotos da artéria cerebral média.
\end{abstract}

PALAVRAS-CHAVE: aneurismas não rotos, artéria cerebral média, microcirurgia.

\begin{abstract}
Surgical treatment of unruptured middle cerebral artery aneurysms
ABSTRACT - Thirty four surgical cases of unruptured middle cerebral artery aneurysms are presented. There was a male prevalence. $83 \%$ of aneurysms was small $(<10 \mathrm{~mm})$. There was a low morbidity related to the surgical treatment. We advise the surgical treatment of unruptured middle cerebral artery aneurysms as first choice to patients with this condition.

KEY WORDS: unruptured aneurysms, middle cerebral artery, microsurgery.
\end{abstract}

Estudos de autópsia tem demonstrado que os aneurismas intracranianos ocorrem na população geral com freqüência que varia de 0,2 a $9,9 \%$, sugerindo que 10 a 15 milhões de pessoas nos Estados Unidos tenham aneurismas intracranianos ${ }^{1}$. Estudos angiográficos reportam a incidência de 3,6 a $6 \%$ na população, com hemorragia subaracnóidea (HSA) em 6 a 8 casos por 100000 habitantes $^{2}$

Esses dados sugerem que existe prevalência de 0,4 a $6 \%$ de aneurismas não rotos ${ }^{3}$, a qual recentemente, com técnicas de diagnóstico mais sofisticadas, vem se mostrando cada vez maior. ${ }^{3}$ Diante deste quadro torna-se importante a decisão da melhor conduta nos aneurismas cerebrais diagnosticados incidentalmente.

0 presente estudo descreve a experiência pessoal do autor sênior no tratamento cirúrgico dos aneurismas não-rotos da artéria cerebral média (ACM), discutindo a história natural, morbimortalidade e indicações de tratamento.

\section{MÉTODO}

Foram selecionados, retrospectivamente, 34 pacientes com idades entre 29 e 75 anos, com 36 aneurismas não rotos da arteria cerebral média (ACM). Eles foram tratados por técnica microcirúrgica, no período de 1971 a 2000, no Hospital Felício Rocho, Belo Horizonte$M G$.
Utilizou-se um protocolo para coleta de dados como se segue: idade: $0-20$; 21-40; 41-60; > 61 anos; sexo; circunstâncias do diagnóstico: aneurismas não rotos sem história de HSA, aneurismas não rotos com história de HSA em outra localização - aneurismas múltiplos; escala de Hunt Hess à admissão ; presença ou ausência de déficit neurológico prévio ; tamanho dos aneurismas $(0-10 \mathrm{~mm}$; 11-25mm e >25mm); clipagem: (total ou parcial); condições de alta (normal ; déficit leve; déficit moderado; dependente; óbito); avaliação pós-operatória de 1 ano (normal; déficit leve; moderado; dependente; óbito).

Este estudo foi aprovado pela Comissão de Ética deste Hospital.

\section{RESULTADOS}

De um total de 820 aneurismas, em 798 pacientes, 20\% foram da ACM ( $n=170), 36$ sendo aneurismas não-rotos da ACM , correspondendo a $21 \%$ dos aneurismas da ACM , e $4 \%$ de todos os aneurismas tratados cirurgicamente. 0 sexo masculino foi o mais acometido com $59 \%$ dos aneurismas nãorotos da ACM, sendo a faixa etária prevalente a de 41-60 anos, $50 \%$ dos casos, seguido pela faixa de $21-40$ anos, $32 \%, e>60$ anos, $18 \%$.

Oitenta e cinco por cento dos aneurismas foram achados incidentais em estudos de imagem em que se utilizou tomografia computadorizada (TC) ou ressonância magnética

Serviço de Neurocirurgia do Hospital Felício Rocho, Belo Horizonte MG, Brasil: ${ }^{1 P r e c e p t o r ~ C h e f e ~ d a ~ r e s i d e ̂ n c i a ~ d e ~ n e u r o c i r u r g i a ; ~}{ }^{2}$ Residente de neurocirurgia; ${ }^{3}$ Neurocirurgião.

Recebido 11 Abril 2003, recebido na forma final 8 Outubro 2003. Aceito 26 Novembro 2003.

Dr. Newton José Godoy Pimenta - Hospital Felício Rocho - Avenida do Contorno 9530 - 30110-130 Belo Horizonte MG - Brasil. E-mail : newtongodoy@terra.com.br 
(RM) ou mediante estudo angiográfico. Os demais aneurismas não-rotos daACM foram diagnosticados devido a sangramento prévio de aneurismas em outra localização.

0 tamanho mínimo do aneurisma neste estudo foi $4 \mathrm{~mm}$ e 0 máximo $28 \mathrm{~mm}$. Oitenta e três porcento apresentaram tamanho entre $0-10 \mathrm{~mm}$, sendo menos freqüente os aneurismas entre $11-25 \mathrm{~mm}, 8,5 \%$, e os de $25 \mathrm{~mm}, 8,5 \%$.

À admissão, todos os pacientes apresentavam-se em Hunt Hess 0 e apenas 1 com déficit neurológico leve, sendo este decorrente de evento isquêmico relacionado à estenose de carótida extracraniana.

Os aneurismas foram clipados totalmente com boa visibilização de seu colo, segundo impressão per-operatória. Não foram realizadas angiografias de controle para se avaliar o bom posicionamento do clipe.

À alta, $8 \%$ dos pacientes apresentaram déficit neurológico leve. Não houve óbito na presente série. Após avaliação de 1 ano a morbidade foi $3 \%$, com boa recuperação dos demais pacientes. Não ocorreram ressangramentos neste período.

\section{DISCUSSÃO}

Em função do avanço tecnológico dos métodos propedêuticos, a incidência de aneurismas cerebrais incidentais vem aumentando, impondo ao neurocirurgião uma decisão terapêutica. Quais aneurismas devem ser tratados? E, se devem ser tratados, qual o tratamento adequado? Vários estudos investigaram a história natural dos aneurismas intracranianos não-rotos, tentando determinar fatores que poderiam predizer uma ruptura subseqüente. Yasui e col. ${ }^{4}$, avaliaram 360 pacientes que foram tratados clinicamente de abril de 1969 a dezembro de 1992 , com seguimento de 234 pacientes $(65 \%)$. Desses pacientes, 34 apresentaram quadro de HSA $(14,5 \%)$, fatal em 18 casos (7,7\%). 0 risco anual de ruptura foi 2,3\%. 0 risco cumulativo de sangramento para todos os pacientes foi $20 \%$ em 10 anos e $35 \%$ em 15 anos. A probabilidade de ruptura foi maior naqueles com aneurismas múltiplos. Outro estudo ${ }^{1}$ separou os pacientes com história de HSA daqueles sem relato desse quadro. No caso de pacientes sem história de sangramento e aneurismas $<10 \mathrm{~mm}, 0$ risco anual de sangramento foi $0,05 \%$, ao passo que aneurismas do mesmo tamanho em pacientes com relato de sangramento prévio tiveram risco 11 vezes maior ( $0,5 \%$ ao ano). Nos pacientes com aneurismas $>10 \mathrm{~mm}$ o risco de ruptura foi 20 vezes maior (1\% ao ano). Nesse estudo, o tamanho e a localização dos aneurismas foram fatores independentes para 0 sangramento, com risco 4,4 vezes maior de ruptura dos aneurismas da circulação posterior se comparado aos da anterior. No Estudo Cooperativo de Aneurismas Intracranianos e Hemorragia Subaracnóidea, que envolveu 6038 aneurismas rotos, o tamanho crítico para ruptura foi 7 a $10 \mathrm{~mm}^{5}$. Em outro estudo, houve risco cumulativo de sangramento em torno de $10,5 \%$ em 10 anos, $23 \%$ em 20 anos e $30,3 \%$ em 30 anos ${ }^{6}$. Tendo em vista os dados atuais, a história natural dos aneurismas depende de algumas de suas características, tais como tamanho e localização, embora não se tenha conseguido definir com precisão o tamanho crítico do aneurisma para se indicar o tratamento preventivo.

Dois são os principais métodos que podem ser utilizados na terapêutica dos aneurismas não-rotos, a microcirurgia e 0 tratamento endovascular.

0 tratamento cirúrgico é a técnica mais consagrada. Vários estudos na literatura, randomizados, meta-analíticos e com longo tempo de seguimento, que avaliam sua morbimortalidade ${ }^{1,7-9}$.

King e col. ${ }^{7}$, em estudo meta-analítico com 733 pacientes, obtiveram índices de 4,1 e $1 \%$ respectivamente para morbidade e mortalidade do tratamento cirúrgico. Raayamakers e col. ${ }^{8}$ observaram 2,6\% de mortalidade e $10,9 \%$ de morbidade. No Estudo Internacional de Aneurismas Não-Rotos ${ }^{1}$, quando os riscos de morbidade e mortalidade foram avaliados levandose também em consideração a idade, observaram-se valores de $6,5 \%$ para pacientes com idade inferior a 45 anos e de $30 \%$ em pacientes com idade acima de 64 anos. Gilvy e Carter ${ }^{9}$ estratificaram a morbidade e mortalidade do tratamento cirúrgico levando em consideração a idade, o tamanho e a localização. 0 risco foi $1 \%$ em pacientes jovens, $5 \%$ em pacientes idosos com aneurismas de circulação anterior maiores que $10 \mathrm{~mm}$ e 15\% nesta mesma faixa etária e tamanho, mas para aneurismas localizados na circulação posterior.

0 tratamento endovascular é técnica mais recente. Estudos reportam índices de morbidade de 4 a 10\% e de mortalidade de $0,5-1 \%$, menores que 0 do tratamento cirúrgico ${ }^{10-12} \cdot 0$ que se questiona na literatura sobre a abordagem endovascular é 0 pequeno seguimento dos pacientes tratados. 0 índice de oclusão total dos aneurismas é 52 a $78 \%{ }^{13}$, com presença de colo residual, em vista da angiografia inicial em $42,4 \%$. Os aneurismas residuais, ocorrem em 4,8\% ${ }^{14}$. A oclusão incompleta pode acarretar ressangramento ${ }^{15,16}$, com incidência anual de $0,5 \%{ }^{13}$, índice este correspondente a história natural desses aneurismas. ${ }^{1}$.

É opinião dos autores que a técnica microcirúrgica para o tratamento dos aneurismas, diferente do tratamento endovascular, permite a confirmação per-operatória da clipagem, além de possuir seguimento longo, dispensado portanto a realização de angiografia pós-operatória e sua morbidade associada. Mais importante do que decidir qual a melhor técnica a ser utilizada no tratamento, talvez seja definir quais os pacientes em que indicaremos tratamento. A literatura não é muito conclusiva em afirmar quais os pacientes devem ser tratados.

Em nosso estudo, encontramos como faixa etária prevalente para ocorrência de aneurismas não-rotos a situada entre 4160 anos, predominando no sexo masculino, sendo $83 \%$ dos aneurismas menores que $10 \mathrm{~mm}$, observando-se morbidade 
cirúrgica inicial de 8\% e, após 1 ano, de 3\% e nenhum óbito.

Esses dados, corroborados por dados da literatura, indicam um baixo índice de morbidade e mortalidade do tratamento cirúrgico dos aneurismas não-rotos, se comparado à evolução devastadora de uma HSA. ${ }^{17}$. Alguns estudos sugerem apenas o tratamento de aneurismas não-rotos maiores que $10 \mathrm{~mm}^{1}$, outros relatam que aneurismas se rompem com maior freqüência entre 7-10 mm5 , sendo ainda sugerido o tratamento cirúrgico de aneurismas não-rotos menores que $10 \mathrm{~mm}^{18-20}$. Outros estudos têm demonstrado, também, que essa diferença no tamanho crítico para ruptura entre aneurismas nãorotos e rotos se encontra na constituição de sua parede, o que permite ao primeiro maior resistência ${ }^{21,22}$. Para os autores, todos os aneurismas vistos em estudo angiográfico devem ser tratados, tendo em vista a incapacidade dos métodos propedêuticos em diferenciar a espessura da parede deles e predizer se eles serão ou não aneurismas mais resistentes ao sangramento. 0 diâmetro do aneurisma não deve ser decisivo, pois o importante para a ruptura é a espessura da parede. Em nossa experiência com aneurismas que sangram, $80 \%$ tinham diâmetro menor que $10 \mathrm{~mm}$ e provocaram óbito ou lesões cerebrais devastadoras por hematoma ou vasoespasmo. Elas poderiam ser prevenidas pelo tratamento precoce.

Concluindo, a técnica, microcirúrgica para abordagem dos aneurismas não-rotos da ACM propicia morbidade e mortalidade aceitáveis e elevado índice de oclusão do colo dos aneurismas. Este é o principal diferencial em relação ao tratamento endovascular, estando de acordo com Regli e col. ${ }^{23}$. Quanto à idade dos pacientes e o tamanho dos aneurismas, embora alguns estudos estabeleçam limite para o tratamento cirúrgico ${ }^{1,23}$, o mais importante é a individualização, devendose levar em consideração outras comorbidades, facilidade do acesso cirúrgico e a experiência do cirurgião.

\section{REFERÊNCIAS}

1. International Study of Unruptured Intracranial Aneurysms Investigators. Unruptured intracranial aneurysms, risk of rupture and risks of surgical intervention. N Eng J Med 1998; 3339:1725-1733.

2. Wardlaw JM, White P M. The detection and management of unruptured intracranial aneurysms. Brain 2000;123:205-221.

3. Rinkel GJ, Djibuti M, van Gijn J. Prevalence and risk of rupture of intracranial aneurysms: a systematic review. Stroke 1998;29:251-256.

4. Yasui N, Suzuki A, Nishimura H, Suzuki K, Abe T. Longterm followup study of unruptured intracranial aneurysms. Neurosurgery 1997;40:1155-1159.

5. Locksley HB. Natural history of subarachinoid hemorrage, intracranial aneurysms and arteriovenous malformations. J Neurosurg 1966;25:321-368.

6. Juvela S, Porras M, Poussa K. Natural history of unruptured intracranial aneurysms: probability of and risk for aneurysm rupture. J Neurosurg 2000;90:379-387.

7. King JT Jr, Berlin JA, Flamm ES. Morbity and mortality from elective surgery for asymptomatic, unruptured, intracranial aneurysms: a metaanalysis. J Neurosurg 1994;81:837-842.

8. Raaymakers TW, Rinkel GJ, Limburg M, Algra A. Mortality and morbity of surgery for unruptured intracranial aneurysms: a meta-analysis. Stroke 1998;29:1531-1538.

9. Ogilvy CS, Carter BS. Stratification of outcome for surgically treated unruptured intracranial aneurysms. Neurosurgery 2003;52:82-88.

10. Johnston SC, Zhao S, Dudley RA, Berman MF, Gress DR. Treatment of unruptured cerebral aneurysms in California. Stroke 2001;32:397-605.

11. Johnston SC, Dudley A, Gress DR, Ono L. Surgical and endovascular treatment of unruptured cerebral aneurysms at university hospital. Neurology 1999;52:1799-1805.

12. Brilstra EH, Rinkel GJ, van der Graaf Y, van Rooij WJ, Algra A. Treatment of intracranial aneurisms by embolizations with coils: a systematic review. Stroke 1999;30:470-476.

13. Vinuela F, Duckwiler G, Maward M. Guglielmi detachable coil embolization of acute intracranial aneurysms: perioperative anatomical and clinical outcome in 403 patients. J Neurosurg 1997;86:475-482.

14. Roy D, Milot G, Raymond J. Endovascular treatment of unruptured aneurysms. Stroke 2001;32:1998-2004.

15. Hodgson TJ, Carroll T, Jellinek DA. Subaracnoid hemorrhage due to late recurrence of a previously unruptured aneurysms after complete endovascular occlusion. Am J Neuroradiol 1998;19:1939-1941.

16. Conrad MD, Guyotat IP, Morel C, Madarassy G, Schonauer C, Deruty R. Estudo comparativo entre aneurismas rotos tratados por cirurgia e por via endovascular. Arq. Neuropsiquiatr 2002;60:96-100.

17. Orz YI, Hongo K, Tanaka Y, et al. Risks of surgery for patients with unruptured intracranial aneurysms. Surg Neurol 2000;53:21-27.

18. Kasuya H, Onda H, Takeshita M, Hori T, Takakura K. Clinical features of intracranial aneurysms in sibling. Neurosurgery 2000;46:1301-1305.

19. Orz YI, Kobayashi S, Tanaka Y. Aneurysms size: a prognostic factor for rupture. Br J Neurosurgery 1997;11:144-149.

20. Rosenorn J, Eskesen V. Does a safe size-limit exist for unruptured intracranial aneurysms? Acta Neurochir 1993;121:113-118.

21. Nawashiro H. Aneurysmal walls in unruptured intracranial aneurysms. Lancet 2000;15;355:1368.

22. Kataoka K, Taneda M, Asai T, Yamada Y. Difference in nature of ruptured and unruptured cerebral aneurysms. Lancet 2000;355:203.

23. Regli L, Uske A, de Tribolet N. Endovascular coil placement compared with surgical clipping for the treatment of unruptured middle cerebral artery aneurysms: a consecutive series. J Neurosurg 1999;90:1025-1030. 